Revista Brasileira de Meteorologia, v.28, n.3, 317 - 330, 2013

\title{
AVALIAÇÃO DE DESEMPENHO DOS MODELOS DO CMIP5 QUANTO À REPRESENTAÇÃO DOS PADRÕES DE VARIAÇÃO DA PRECIPITAÇÃO NO SÉCULO XX SOBRE A REGIÃO NORDESTE DO BRASIL, AMAZÔNIA E BACIA DO PRATA E ANÁLISE DAS PROJEÇÕES PARA O CENÁRIO RCP8.5
}

\author{
CLEITON DA SILVA SILVEIRA ${ }^{1}$, FRANCISCO DE ASSIS DE SOUZA FILHO ${ }^{1}$, ALEXANDRE \\ ARAUJO COSTA ${ }^{2}$, SAMUELLSON LOPES CABRAL ${ }^{1}$
}

\author{
${ }^{1}$ Universidade Federal do Ceará, Departamento de Engenharia Hidráulica e Ambiental (UFC/DEHA), \\ Fortaleza, CE, Brasil \\ ${ }^{2}$ Universidade Estadual do Ceará (UECE), Mestrado em Ciências Físicas Aplicada, Fortaleza, CE, Brasil
}

cleitonsilveira16@yahoo.com.br, assis@ufc.br, alexandrearaujoc@gmail.com, samuellsoncabral@hotmail.com

Recebido Setembro de 2011 - Aceito Janeiro de 2013

\begin{abstract}
RESUMO
Os modelos globais do Coupled Model Intercomparison Project Phase 5 (CMIP5) são avaliados para a região Nordeste do Brasil (NEB), região Amazônica e bacia do Prata quanto à representação da precipitação para o período de 1901 a 1999. Além disso, são analisadas as projeções de precipitação para o cenário RCP8.5 para o século XXI. A avaliação é realizada utilizando-se os dados do Climatic Research Unit (CRU) e a reanálise 20th Century Reanalysis V2 do National Oceanic and Atmospheric Administration (NOAA). Os modelos são classificados através de índices que indicam como os padrões de variação sazonal, interanual e decenal são representados. A avaliação apontou como melhores modelos para o NEB as rodadas do modelo CANESM, enquanto para a bacia do Prata, a rodada do modelo francês CNRM_CM5_r1ilp1. Já para a Amazônia, destacam-se as rodadas do modelo GISS-E2-R. Na região NEB, a maioria dos modelos mostra maiores impactos na pré-estação, porém divergem quanto ao sinal da anomalia. Na região Amazônica, os modelos sugerem maiores possibilidades de redução na precipitação, em até 20,5\%,33,6 e 39,5\% para os períodos de 2010 a 2039, 2040 a 2069 e 2070 a 2099, respectivamente. Na região do Prata, o conjunto dos modelos projeta poucas alterações no período de 2010 a 2039.
\end{abstract}

Palavras-chave: Mudanças Climáticas, CMIP5, América do Sul, RCP8.5.

ABSTRACT: PERFORMANCE ASSESSMENT OF CMIP5 MODELS CONCERNING THE REPRESENTATION OF PRECIPITATION VARIATION PATTERNS IN THE TWENTIETH CENTURY ON THE NORTHEAST OF BRAZIL, AMAZON AND PRATA BASIN AND ANALYSIS OF PROJECTIONS FOR THE SCENERY RCP8.5

The global models of the Coupled Model Intercomparison Project Phase 5 (CMIP5) are evaluated over Northeast Brazil (NEB), the Amazon region and La Plata Basin regarding the representation of precipitation for the period 1901 to 1999. Furthermore, the projections of precipitation for scenario RCP8.5 for the XXI century are analyzed. The evaluation is performed using data from the Climatic Research Unit (CRU) and 20th Century Reanalysis V2 reanalysis of the National Oceanic and Atmospheric Administration (NOAA). The models are classified using indices that indicate how patterns of seasonal, interannual and decennial variation are represented. The evaluation identified CANESM as the best models for NEB, whereas for La Plata Basin, the French model CNRM_CM5 r1ilp1 runs were superior. For the Amazon region, the model GISS Model-E2-R_r1ilp1 exhibited the best performance. Over NEB, most models indicate largest changes during the pre-season, but differ on the sign of the anomaly. In the Amazon region the models suggest greater chances of rainfall reduction up to $20.5 \%, 33.6$ and $39.5 \%$ for the periods $2010-2039,2040-2069$ and 2070-2099, respectively. Over the La Plata region, the, ensemble projects few changes for the 2010 to 2039 period .

Keywords: Climate Change, CMIP5, América do Sul, RCP8.5. 


\section{INTRODUÇÃO}

O Intergovernmental Panel on Climate Change (IPCC) é o principal órgão científico internacional para as avaliações das alterações climáticas, e foi criado pelo Programa das Nações Unidas para o Ambiente (PNUA) e pela Organização Mundial de Meteorologia (OMM) em 1988, para fornecer visões científicas claras sobre o estado atual do conhecimento das mudanças climáticas e seus possíveis impactos sócio-econômicos e ambientais (IPCC, 2007).

Desde 1988, quando foi criado IPCC, cientistas vinculados aos principais centros de pesquisa em clima reúnem-se para publicar relatórios sobre os possíveis cenários de emissão de gases de efeito estufa na atmosfera e os impactos nas diversas variáveis climáticas. Até o momento, foram publicados quatro relatórios: em 1990 em Sundsvall (Suécia), 1995 em Roma (Itália), 2001 em Acra (Gana) e 2007 em Paris (França).

O quarto relatório fornecido pelo IPCC, divulgado em fevereiro de 2007, confirma a projeção de aumento da temperatura média da atmosfera. Dados evidentes de mudanças globais foram apresentados no AR4, em decorrência do aumento da concentração dos gases de efeito estufa a partir de atividades humanas. As concentrações de dióxido de carbono $\left(\mathrm{CO}_{2}\right)$, de gás metano $\left(\mathrm{CH}_{4}\right)$ e de óxido nitroso $\left(\mathrm{N}_{2} \mathrm{O}\right)$ ultrapassaram em muito os valores pré-industriais. Os indicadores de mudanças climáticas mostrados por esse relatório foram o aumento da temperatura global, aumento do nível do mar e a redução da cobertura de gelo. (IPCC, 2007).

A mudança na concentração desses gases tem causado um evidente aumento gradual da temperatura global (IPCC 2007), e pode alterar outros campos meteorológicos, como a precipitação. Esse aumento de temperatura é mostrado para o Brasil para o século XX em, por exemplo, Victoria et al. (1998) e Duursma (2002), Nobre (2005) .

Embora exista uma discrepância em torno dos valores absolutos dos modelos do Coupled Model Intercomparison Project Phase 3 (CMIP3) para o aumento na temperatura, todos concordam que haverá aumento na temperatura média global (IPCC, 2007). Entretanto, os diferentes modelos do CMIP3, publicados pelo IPCC-AR4 mostraram cenários divergentes para o campo de precipitação para várias regiões do planeta, alguns projetando aumento de precipitação e outros, redução (Marengo e Valverde, 2007; Marengo e Soares, 2005).

Diversos autores avaliaram a tendência na precipitação total para séries observadas durante o século XX. Por exemplo, Haylock et al. (2006) fizeram uma análise da precipitação sobre a América do Sul, e observaram uma tendência de aumento do total anual de chuva sobre o Nordeste do Brasil (NEB). O estudo realizado por Santos e Britto (2007), utilizando índices de extremos climáticos e correlacionados com as anomalias de temperatura da superfície do mar (TSM), também mostrou tendência de aumento da precipitação total anual nos Estados da Paraíba e Rio Grande de Norte. Costa dos Santos et al. (2009) mostraram tendência de aumento de precipitação para o Estado do Ceará.

Entretanto, tais tendências podem estar associadas, além de mudanças climáticas e/ou alterações no uso do solo, a flutuações naturais dessa grandeza que podem ocorrer em várias escalas de tempo, incluindo alguns anos (variabilidade interanual), décadas (variabilidade inter e multidecadal) e até em escalas de tempo mais longas, relevantes em estudos paleoclimáticos. Essas flutuações podem estar ligadas, por exemplo, a padrões de variabilidade nos Oceanos Pacífico e Atlântico (Grimm et al., 2000, Kane, 1992), como o fenômeno El Niño Oscilação Sul (ENSO, na escala de tempo interanual), ou à variabilidade decadal do Pacífico. No entanto, além das variações naturais, é provável que a alteração da temperatura planetária das últimas décadas causada pela elevação da concentração de gases estufas por emissões antrópicas (IPCC, 2007), provoque mudanças no regime de chuvas na América do Sul.

Espera-se que algumas das questões científicas, que surgiram durante a preparação do quarto relatório do IPCC, possam ser respondidas com base no conjunto de dados do Coupled Model Intercomparison Project Phase 5 (CMIP5), ao subsidiar a publicação do Quinto Relatório (AR5, prevista para publicação no final de 2013).

Entretanto, apesar do significativo desenvolvimento ocorrido nas últimas décadas acerca do conhecimento do clima, existem ainda diversas incertezas associadas aos diversos sistemas de previsões ou projeções climáticas. Por exemplo, as parametrizações físicas, método numérico de resolução adotado, as condições iniciais e fronteira e a própria atmosfera que é considerada um sistema caótico (Lorenz, 1965).

Quanto às questões relacionadas aos modelos globais de mudanças climáticas do CMIP5, pode se adicionar ainda a baixa resolução dos modelos que não é capaz de reproduzir alguns processos físicos e a trajetória desconhecida de emissões de gases de efeito estufa no futuro. Dado o grande número de modelos e as projeções tão divergentes, é imprescindível uma metodologia de avaliação do comportamento dos modelos, que seja capaz de identificar aqueles que representam adequadamente a região de interesse. Esta avaliação pode ser vista por duas vertentes: a primeira consiste em identificar os melhores modelos e admitir que uma melhor representação do século XX levaria a uma representação mais coerente do futuro; já a segunda consiste em identificar os piores modelos e retirálos do conjunto para posterior análise estatística.

Segundo Albuquerque et al. (2009), a América do Sul apresenta clima com intensa variabilidade temporal e espacial de chuvas, devido à atuação de diversos sistemas. 
Essa complexidade torna a região vulnerável às condições de tempo e clima, que são associadas a impactos sociais e econômicos significativos em diversos setores. Desta forma, torna-se relevante para o desenvolvimento de políticas públicas a identificação das alterações na ocorrência do clima nesta região e os possíveis impactos no regime de chuvas.

O objetivo do presente estudo é desenvolver uma metodologia de avaliação da habilidade dos modelos do CMIP5 de prever o regime sazonal, interanual e interdecadal de chuvas e aplica-las a regiões específicas sobre a América do Sul. Além disso, pretende-se avaliar as projeções do cenário RCP8.5 dos modelos do CMIP5 para o século XXI.

\section{METODOLOGIA}

\subsection{Região de estudo}

Na Figura 1, apresenta-se a localização espacial das três grades que englobam as regiões de estudo: a do Nordeste Setentrional Brasileiro (grade 1), compreendida entre as coordenadas $0^{\circ}$ a $10^{\circ} \mathrm{S}$ de latitude e $33^{\circ} \mathrm{O}$ a $44^{\circ} \mathrm{O}$ de longitude, da Amazônia (grade 2), entre as coordenadas $3,5^{\circ} \mathrm{N}$ a $12^{\circ} \mathrm{S}$ de latitude $49^{\circ} \mathrm{O}$ a $75,5^{\circ} \mathrm{O}$ de longitude e do Prata (grade 3 ) compreendida entre $16,5^{\circ} \mathrm{S}$ a $36^{\circ} \mathrm{S}$ de latitude e longitude $44^{\circ}$ $\mathrm{O}$ a $64^{\circ} \mathrm{O}$, respectivamente.

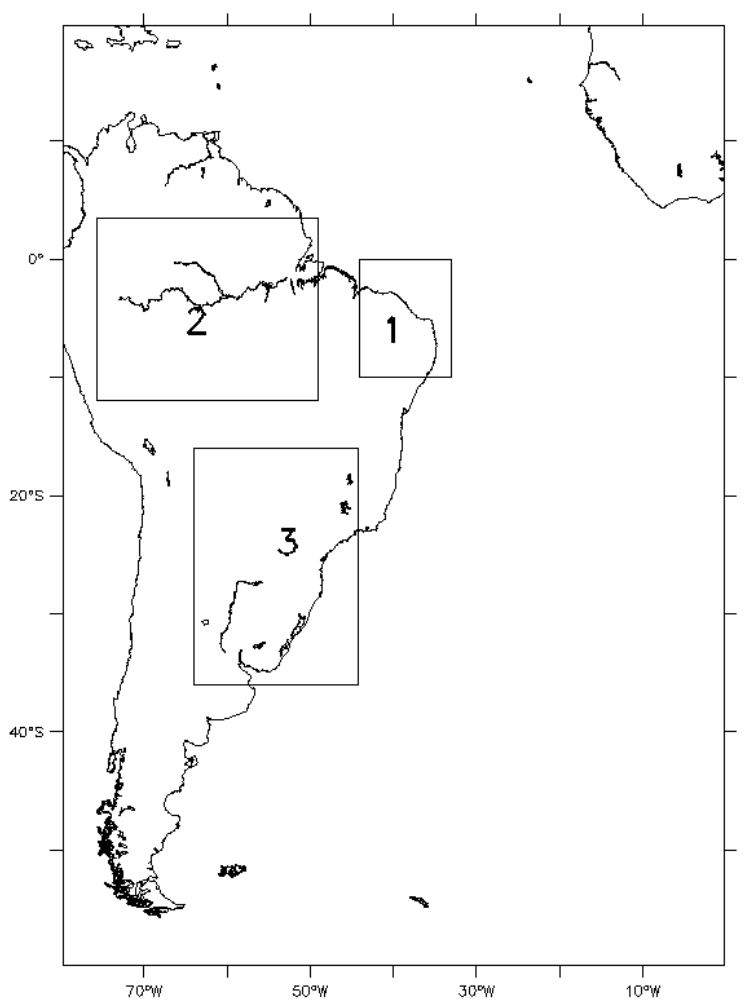

Figura 1 - Localização das regiões estudadas: Nordeste Setentrional brasileiro (1), Amazônia (2) e bacia do Prata (3).

\subsection{Dados observacionais}

A base de dados observacionais utilizada para verificar a destreza dos modelos do IPCC sobre o continente são provenientes da University of East Anglia/Climate Research Unit (CRU) (New et. al, 1999 e New et. al, 2001) (http://badc. nerc.ac.uk/data/cru/). Enquanto na porção da grade sobre o oceano são usadas as reanálises 20th CenturyReanalysis V2 (Compo, 2006; Whitaker, 2004)(NOAA/OAR/ESRL PSD, Boulder, Colorado, EUA, a partir do seguinte site: http://www. esrl.noaa.gov/psd/) fornecidos pelo National Oceanic and Atmospheric Administration (NOAA).

O conjunto de dados utilizado corresponde à climatologia de precipitação de 1901 a 1999 , com resolução de $0,5^{\circ}$ para o continente (CRU TS 3.0) e 2,0 graus para oceano (20th Century Reanalysis V2).

\subsection{Modelos do CMIP5}

Os dados provenientes do CMIP5 são resultados de simulações de modelos globais de alguns centros de pesquisa que contribuem para confecção dos relatórios dos IPCC (conforme Tabela 1), forçadas pelas concentrações observadas de gases de efeito estufa durante o século XX. Para cada modelo foram considerados diferentes membros, conforme disponibilizados pelo CMIP5. O código das configurações das simulações disponibilizadas é apresentado na forma "rxiypz", tal que:

- r = "realizações", isto é, "rodadas", com a mesma física e condições iniciais diferentes;

- $\mathrm{i}=$ inicializações diferentes, com implicações físicas;

- $\mathrm{p}$ = perturbação na física.

Ou seja, a simulação que apresentar o seguinte código: model_r1ilp1 significa que se trata da "rodada" 1, com inicialização 1 e física 1 do modelo "model". O caso rlilp1 de cada modelo será chamado, no texto, de run1.

Como parte da fase preparatória para o desenvolvimento dos novos cenários para o AR5, foram criados os chamados RCPs (Representative Concentration Pathways), que servem como entrada para modelagem climática e química atmosférica nos experimentos numéricos do CMIP5. Os RCPs recebem seus nomes a partir dos níveis das forçantes radiativas, conforme relatado por cada equipe de modelagem elaboradora de cada RCP. Assim, RCP-X implica em um cenário no qual a forçante radiativa de estabilização ou de pico ou ao final do século XXI corresponde a X W. $\mathrm{m}^{-2}$. Na Figura 2 é mostrado o comportamento dos principais cenários do IPCC-AR5: RCP8.5, RCP6, RCP4.5 e RCP3-PD. Neste trabalho foi usado o cenário RCP8.5 para análise das projeções do século XXI. 
Tabela 1 - Modelos do CMIP5 analisados.

\begin{tabular}{cc}
\hline Modelo & Instituição \\
\hline HadGEM2-ES & Met Office Hadley Centre \\
GISS-E2-H & NASA Goddard Institute for Space Studies \\
GISS-E2-R & NASA Goddard Institute for Space Studies \\
IPSL-CM5A-LR & Institut Pierre-Simon Laplace \\
CCCMA/CanESM2 & Canadian Centre for Climate Modelling and Analysis \\
CNRM-CM5 & Centre National de Recherches Meteorologiques/ Europeen de Recherche et Formation Avancees en Calcul \\
& Scientifique (CNRM/CERFACS) \\
INM_CM4 & Institute of Numerical Mathematics, Russian Academy of Science
\end{tabular}

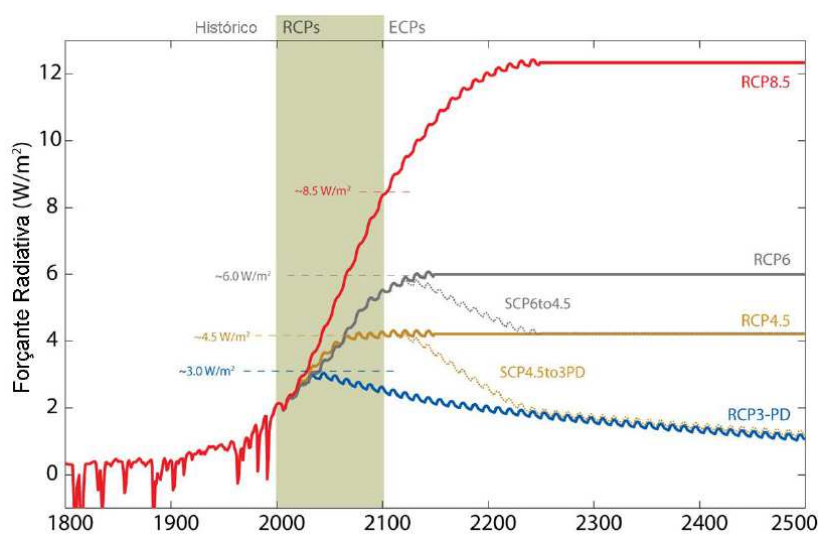

Figura 2 - Cenários do IPCC-AR5 e forçante radiativa antropogênica global para cada um deles.

Fonte: http://www.pik-potsdam.de/ mmalte/rcps/.

\subsection{Critérios de avaliação geral}

Para a avaliação geral dos modelos do CMIP5, é proposto o modelo hierárquico da Figura 3.

Em busca de identificar os modelos que melhor representam os padrões de variação do século XX, foi adotada a avaliação sazonal proposta por Silveira et al.(2012) e uma adaptação da avaliação plurianual proposta por Lázaro et al. (2011).

A avaliação foi constituída por três etapas que analisam padrões de variação de diferentes escalas: sazonal, interanual e interdecadal.

Para avaliação sazonal $\left(\mathrm{AVAL}_{\mathrm{s}}\right)$ dos modelos, foi calculada a climatologia mensal média sobre a região de estudo para todos os modelos do IPCC e a observação (CRU continente e NOAA oceano). Em seguida foi feita uma comparação com base em alguns índices estatísticos para que possam ser definidos quais modelos possuem melhor comportamento para América do Sul.
As avaliações interanual $\left(\mathrm{AVAL}_{\mathrm{a}}\right)$ e interdecadal $\left(\mathrm{AVAL}_{\mathrm{d}}\right.$ ) baseiaram-se na transformada de ondeletas (Torrence e Compo, 1998), sobretudo na comparação do espectro global de variação das séries observadas e modeladas.

Cada uma das avaliações possui valores entre zero e 1, que representam, respectivamente, o pior e o melhor modelo para todos os índices daquela análise. Depois de calculados $\mathrm{AVAL}_{\mathrm{s}}, \mathrm{AVAL}_{\mathrm{a}}$ e $\mathrm{AVAL}_{\mathrm{d}}$ de todos os modelos foi sugerido um índice geral, $\mathrm{AVAL}_{\mathrm{t}}$, conforme Equações 1 e 2. Este é dado pelo somatório das avaliações, ponderados por um valor $\gamma_{\mathrm{j}}$ (que possui valores entre zero e 1). Esta ponderação pode ser feita de acordo com os interesses do avaliador: ao atribuir valores iguais para $\gamma_{\mathrm{j}}$, todas as avaliações possuem o mesmo peso no índice geral, enquanto ao atribuir valores diferentes, as avaliações têm pesos distintos na análise gera:

$$
\begin{aligned}
& A V A L_{G}=\sum_{j=1}^{n} \gamma_{j} \cdot A V A L_{j} \\
& \sum_{j=1}^{n} \gamma_{j}=1
\end{aligned}
$$

\subsubsection{Critérios de avaliação do desempenho dos modelos do IPCC quanto à representação da sazonalidade}

Utilizou-se como critério de avaliação a representação da sazonalidade das precipitações, que é de grande relevância para a avaliação dos impactos do clima em recursos hídricos e agricultura, afinal o início do plantio das culturas e o regime fluvial são condicionados pela distribuição temporal das chuvas. Uma má representação dessa sazonalidade pode comprometer seriamente a avaliação dos impactos das mudanças climáticas sobre estas duas áreas importantes. Adicionalmente, pode-se avaliar que os totais mensais de precipitação e sua sazonalidade são considerados como indicativo da qualidade que o modelo tem de representar os sistemas geradores de chuvas e sua ocorrência. 


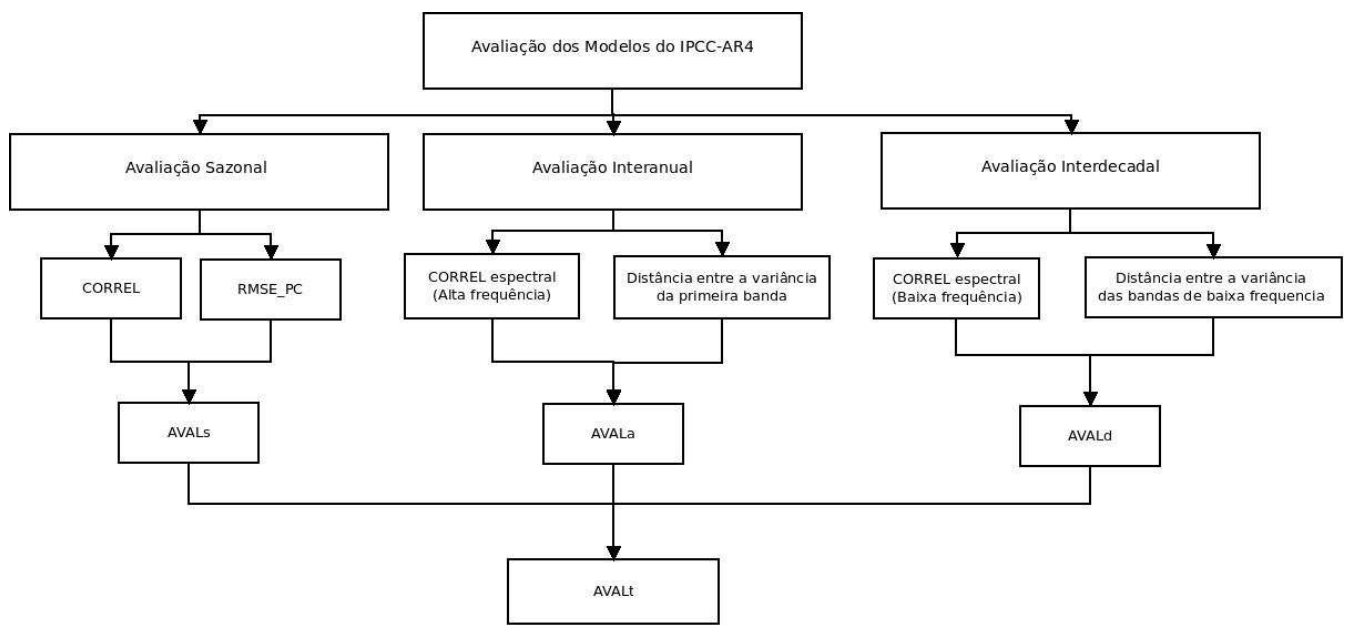

Figura 3 - Critérios de avaliação dos modelos do CMIP5 no período de 1901 a 1999.

Para avaliação sazonal dos modelos foi calculada a climatologia mensal média sobre a região de estudo para os modelos dos CMIP5 enumerados na Tabela 1 e as observações (CRU continente e NOAA oceano, sem interpolação). Em seguida, foi feita uma comparação com base em alguns índices estatísticos para que possam ser definidos quais modelos apresentam melhor comportamento para as diferentes regiões da América do Sul mostradas na Figura 1.

As medidas estatísticas utilizadas, cujas definições estão indicadas a seguir, conforme sugerido por Silveira et al. (2012), são a raiz do erro quadrático médio da contribuição percentual mensal em relação às chuvas anuais (RMSE_PC) e a correlação de Pearson (CORREL).

A raiz do erro quadrático médio percentual (RMSE_PC) é a raiz quadrada da média das diferenças individuais quadráticas, entre a contribuição percentual mensal das chuvas modeladas, para os totais anuais e o valor correspondente nas observações, sendo definida pela equação:

$$
\text { RMSE_PC }=\sqrt{\frac{1}{n_{i=1}^{n}} \sum_{i=1}^{n}\left(\frac{100 . P_{i}}{\sum_{j=1}^{n} P_{j}}-\frac{100 . A_{i}}{\sum_{j=1}^{n} A_{j}}\right)^{2}}
$$

onde $n$ são os meses, $P$ é previsão de cada mês e $A$, a observação.

Valores grandes de $R M S E_{-} P C$ representam erros maiores nos campos previstos e valores próximos de zero indicam uma previsão quase perfeita. Elevando ao quadrado o termo da diferença, o RMSE_PC tende a dar maior peso às grandes discrepâncias entre os campos observados e previstos.

A correlação pode assumir valores entre - 1 e 1 que indica, respectivamente, perfeita anticorrelação e perfeita correlação, com a ausência total de correlação correspondendo a um valor nulo. Este índice tem a capacidade de detectar correspondência de fase entre as séries, sendo, por construção, insensível a erros de viés. É dado conforme a Equação 4.

$$
\text { CORREL }=\frac{\sum_{i=1}^{i=n}\left(A_{i}-\bar{A}\right)\left(P_{i}-\bar{P}\right)}{\sqrt{\sum_{i=1}^{i=n}\left(P_{i}-\bar{P}\right)^{2} \sum_{i=1}^{i=n}\left(A_{i}-\bar{A}\right)^{2}}}
$$

Após o cálculo desses dois índices foi calculado um índice de avaliação ponderada para que os modelos possam ser classificados, tal que:

$$
\begin{aligned}
A V A L_{s}= & \alpha_{c}\left(\frac{\text { CORREL-CORREL }}{\text { CORREL }_{M A X}-C O R R E L_{M I N}}\right) \\
& +\alpha_{r}\left(\frac{R M S E_{-} P C_{M A X}-R M S E_{-} P C}{R M S E_{-} P C_{M A X}-R M S E_{-} P C_{M I N}}\right) \\
\alpha_{c}+\alpha_{r} & =1
\end{aligned}
$$

Sendo, CORREL $_{\text {MIN }}$ a menor correlação obtida entre os modelos do IPCC e CORREL $\mathrm{MAX}_{\text {a }}$ máxima correlação, assim como RMSE_PC $\mathrm{MAX}_{\mathrm{e}}$ o máximo erro quadrático médio percentual dos modelos do IPCC e RMSE_PC $\mathrm{MIN}_{\mathrm{N}}$, o mínimo.

As variáveis $\alpha_{\mathrm{c}}$ e $\alpha_{\mathrm{r}}$ assumem valores entre 0 e 1 (conforme Equação 6), para $\alpha_{\mathrm{c}}>\alpha_{\mathrm{r}}$, portanto, a correlação exerce maior influência na avaliação do modelo, enquanto para $\alpha_{\mathrm{r}}>\alpha_{\mathrm{c}}$, o RMSE_PC dos modelos possui maior peso na avaliação. Já se $\alpha_{\mathrm{r}}=\alpha_{\mathrm{c}}=0,5$ as duas métricas utilizadas neste trabalho exercem o mesmo efeito sobre o valor de $\mathrm{AVAL}_{\mathrm{s}}$. Nos casos extremos, ao se considerar $\alpha_{\mathrm{r}}=0$ então $\alpha_{\mathrm{c}}=1$, apenas a correlação será usada na avaliação dos modelos, assim como, se $\alpha_{\mathrm{r}}=1$ então $\alpha_{\mathrm{c}}=0$, apenas o RMSE_PC será usado na avaliação dos modelos. 


\subsubsection{CRITÉRIOS DE AVALIAÇÃO DO DESEMPENHO DOS MODELOS DO CMIP5 QUANTO À REPRESENTAÇÃO DA VARIABILIDADE PLURIANUAL}

\subsubsection{Métodos de análise de séries temporais - Wavelets}

Diante da dificuldade em se caracterizar as variabilidades, tanto de espaço, como de tempo no padrão de chuvas, torna-se necessário utilizar um método de análise adequado na localização temporal das estruturas de multiescalas. $\mathrm{O}$ método usado neste estudo é a Transformada de Wavelets (TW). Este método é recomendado para o estudo de fenômenos transientes, espacialmente heterogêneos e que atuam simultaneamente em várias escalas do escoamento (Torrence e Compo, 1998).

A TW é definida em termos de uma integral de convolução entre o sinal analisado $f(t)$ e uma função wavelet conhecida, expressa por:

$$
\mathrm{C}(\mathrm{a}, \mathrm{b})=\int_{-\infty}^{+\infty} f(t) \psi_{a, b}(t) d t
$$

em que os parâmetros $\mathrm{a}$ e $\mathrm{b}$ variam continuamente em $\mathrm{R}$, com $\mathrm{a} \neq 0$, e

$$
\psi_{a, b}(t)=\frac{1}{\sqrt{a}} \psi\left(\frac{t-b}{a}\right), \quad \mathrm{a} \in \mathrm{R}^{+} \mathrm{e} \mathrm{b} \in \mathrm{R}
$$

Sendo as funções $\psi_{\mathrm{a}, \mathrm{b}}$ chamadas wavelets filhas e são geradas a partir de dilatações e translações da wavelets-mãe $\psi(\mathrm{t})$. A função wavelet considerada para a análise foi a de Morlet, dada pela seguinte expressão:

$$
\Psi_{0}(\eta)=\pi^{-1 / 4} e^{i \omega_{0} \eta} e^{-\eta^{2} / 2}
$$

com $w_{0}=6$ e $\eta=t / s$. Onde: $\omega_{0}$ é a frequência adimensional, que, no caso da ondeleta de Morlet, é igual a seis, de forma a satisfazer a condição de admissibilidade; e $\eta$ é o parâmetro adimensional do tempo.

Sendo $t$ o tempo e $s$ é a escala da wavelet. Esta função é complexa e possui características semelhantes àquelas da serie temporal analisada, tais como, simetria ou assimetria, variação temporal brusca ou suave. $\mathrm{O}$ algoritmo usado foi o desenvolvido por Torrence e Compo (1998).

\subsubsection{Critérios de avaliação}

Utilizou-se como critério de avaliação plurianual a representação da série de dados observacionais de 1901 a 1999 da precipitação, através do espectro de energia global dos totais de precipitações anuais. A avaliação plurianual é dividida em duas: avaliação interanual e avaliação interdecadal.
Para avaliação dos modelos foi calculado o espectro de energia global sobre as regiões de estudo para todas as rodadas dos modelos do IPCC e as observações. Em seguida, foram identificados os principais padrões de variação das séries observadas a partir do Espectro Global da Ondeleta e a partir disto foram executadas as seguintes etapas:

- decomposição do sinal para obter os coeficientes wavelets no domínio transformado. Para o caso da série observada de precipitação nas três regiões avaliadas, a decomposição foi igual à soma das bandas características no século XX e o resíduo. A partir da Equação 10, tem-se:

$$
P(t)=R+\sum_{i=1}^{n} B_{i}
$$

$P(t)$ é a série de precipitação média anual observada; $R$ é o resíduo; $i$ é o número de bandas utilizadas; $\mathrm{B}_{i}$ é a ondeleta que representa a banda $i$.

Cada uma das regiões avaliadas neste trabalho apresentou três bandas. Sendo assim, a primeira banda do espectro observado foi considerada variação interanual e as demais regiões do espectro foram consideradas variação interdecadal.

- análise e processamento dos coeficientes neste domínio;

- reconstrução do sinal a partir dos coeficientes modificados.

O processo de reconstrução foi repetido para os modelos do CMIP5 para as mesmas bandas observadas. Em seguida, foi feita uma comparação com base em alguns índices estatísticos, para que possam ser definidos que modelos possuem melhor desempenho para a região de estudo.

As medidas estatísticas utilizadas, cujas definições estão indicadas a seguir, são a correlação entre a reconstituição da série do modelo e da observação para uma dada banda de frequência (CORREL) e uma "distância" calculada a partir das variâncias das rodadas dos modelos (DIST) nas diferentes bandas espectrais.

Sendo as frações entre a variância espectral da banda e a variância do espectro global da série da chuva anual $f_{P}$ e $f_{A}$, tais que

$$
\begin{gathered}
f_{p}=\frac{V P_{S}}{V P_{G}} \\
f_{a}=\frac{V A_{S}}{V A_{G}}
\end{gathered}
$$

onde $V P_{S}$ é a variância da precipitação modelada na região da banda de interesse sobre a região estudada e $V P_{G}$ a variância de todo o espectro global de potência da série modelada e, analogamente, $V A_{s}$ é a variância da observação na banda de interesse e a $V A_{G}$ a variância do espectro global de ondeletas da observação. 
A distância euclidiana DIST foi calculada a partir das razões entre as variâncias por banda definidas nas Equações 12 e é definida pela equação:

$$
D I S T=\sum_{i=1}^{n}\left(f_{p_{i}}-f\right)_{a_{i}}^{2}
$$

em que $n$ é o número de bandas avaliado e $i$ é um índice que se refere à i-ésima banda.

Ao final do cálculo desses índices, foi feita uma avaliação ponderada para representação interanual e outra para interdecadal, conforme as Equações 13 a 16, a fim de que os modelos possam ser classificados. A avaliação interanual é dada por:

$$
\begin{aligned}
A V A L_{a} & =\beta_{c}\left(\frac{\text { OORREL }_{1}-\text { CORREL }_{M I N 1}}{\text { CORREL }_{M A X 1}-C O R R E L_{M I N 1}}\right) . \\
& +\beta_{d}\left(\frac{D_{M S T} T_{M A X 1}-D I S T_{1}}{D_{\text {IST }}-D I S T_{M I N 1}}\right)
\end{aligned}
$$

tal que:

$$
\beta_{c}+\beta_{d}=1
$$

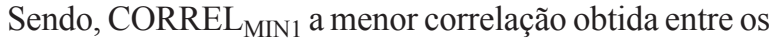
modelos do CMIP5 e CORREL MAX1 $_{1}$ a máxima correlação em relação ao observado na primeira região do espectro avaliada. De modo similar, DIST $\mathrm{MAX}_{1}$ é a o valor máximo da distância entre modelo e observação, referente à variabilidade na banda interanual e $\mathrm{DIST}_{\mathrm{MIN} 1}$, o valor mínimo correspondente.

A avaliação interdecadal é dada por:

$$
\begin{aligned}
A V A L_{d} & =\sigma_{c}\left(\frac{\text { CORREL }_{2}-\text { CORREL }_{M I N 2}}{\text { CORREL }_{M A X 2}-C O R R E L_{M I N 2}}\right) \\
& +\sigma_{d}\left(\frac{\text { IST }_{M A X 2}-\text { IIST }_{2}}{\text { DIST }_{M A X 2}-\text { DIST }_{M I N 2}}\right) \\
\sigma_{c}+\sigma_{d} & =1
\end{aligned}
$$

Sendo CORREL MAX2 $_{2}$, CORREL MIN2 $_{\text {DIST }}$ MAX2 $_{2}$ e DIST $_{\text {MIN2 }}$ referentes à variabilidade interdecadal, com definições análogas às variáveis correspondentes no caso interanual.

As variáveis $\beta_{\mathrm{c}}, \beta_{\mathrm{r}}, \sigma_{\mathrm{c}}$ e $\sigma_{\mathrm{d}}$ assumem valores entre $0 \mathrm{e}$ 1 (conforme Equações 14 e 16). Para valores $\beta_{\mathrm{c}}>\beta_{\mathrm{d}} \mathrm{e} \sigma_{\mathrm{c}}>\sigma_{\mathrm{d}}$, a correlação exerce maior influência na avaliação do modelo, enquanto que para $\beta_{\mathrm{c}}<\beta_{\mathrm{d}}$ e $\sigma_{\mathrm{c}}<\sigma_{\mathrm{d}}$, o índice DIST exerce maior efeito.

As variáveis $\mathrm{AVAL}_{\mathrm{y}}$ e $\mathrm{AVAL}_{\mathrm{d}}$ assumem valores entre 0 e 1 que indicam, respectivamente, o pior entre os modelos avaliados e o melhor deles, segundo o critério adotado.

\subsection{Análise das projeções}

Para o cálculo das anomalias sazonais, foram consideradas as projeções fornecidas pelos modelos globais do CMIP5 para o cenário RCP8.5 no período de 2010 a 2099. Em seguida foi feita uma comparação relativa à representação dos modelos para o século XX no período de 1950 a 1999.

Para o cálculo da anomalia sazonal foram encontradas as climatologias do século XX (simulação histórica) e do século XXI (cenário RCP8.5) para cada um dos modelos globais do CMIP5. Em seguida, foi calculada a diferença entre as mesmas, dividida pela média da precipitação climatológica do século XX naquele mês, conforme a Equação 17:

$$
\mathrm{A}_{\%}^{\mathrm{i}}=\left(\frac{\mathrm{P}_{\mathrm{rcp} 85}^{\mathrm{i}}-\mathrm{P}_{\mathrm{xx}}^{\mathrm{i}}}{\mathrm{P}_{\mathrm{xx}}^{\mathrm{i}}}\right) \cdot 100
$$

onde $i$ são os meses do ano, $\mathrm{P} \_r c p 85^{\wedge} \mathrm{i}$ a precipitação do cenário do século XXI para o mês $i$, a precipitação do século XX para o mês $i$ mostrada pelo modelo e representa a anomalia percentual da precipitação sazonalmente.

Para o cálculo da anomalia na média anual considerou-se a Equação 18:

$$
A_{\text {anual }}=\frac{P_{X X I}^{a}-P_{X X}^{a}}{P_{X X}^{a}} x 100
$$

onde $P_{X X I}^{a}$ é média da precipitação anual para o cenário do século XXI e $P_{X X}^{a}$ é a média da precipitação anual para o cenário do século XXI.

\section{RESULTADOS}

\subsection{Avaliação dos modelos}

\subsubsection{Nordeste setentrional do Brasil}

A Figura 4 mostra as climatologias dos modelos do CMIP5 (apenas a rodada 1) em comparação com as observações sobre a região do Nordeste Setentrional do Brasil, conforme indicado na grade 1 da Figura 1. A maioria dos modelos representa razoavelmente a climatologia observada, porém, principalmente no período da estação chuvosa, os modelos divergem quanto à quantidade de chuva nessa região.

Os modelos GISS_E2R_RUN1, GISS_E2H_RUN1 e INM_CM4_RUN1 superestimam a precipitação climatológica em todos os meses, resultando em totais anuais respectivamente $120 \%, 155 \%$ e $137 \%$ acima do observado. Os modelos GISS_E2H_RUN1 e INM_CM4_RUN1 apresentam erros na distribuição sazonal média da precipitação, subestimando a contribuição percentual das chuvas no primeiro semestre para o total anual e superestimando-a no segundo. O modelo HADGEM2_ES_RUN1 superestima em cerca de 30\% a 


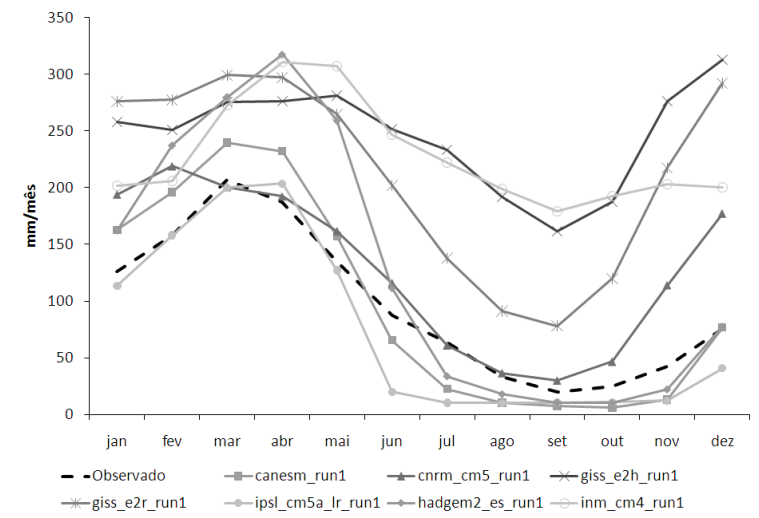

a)

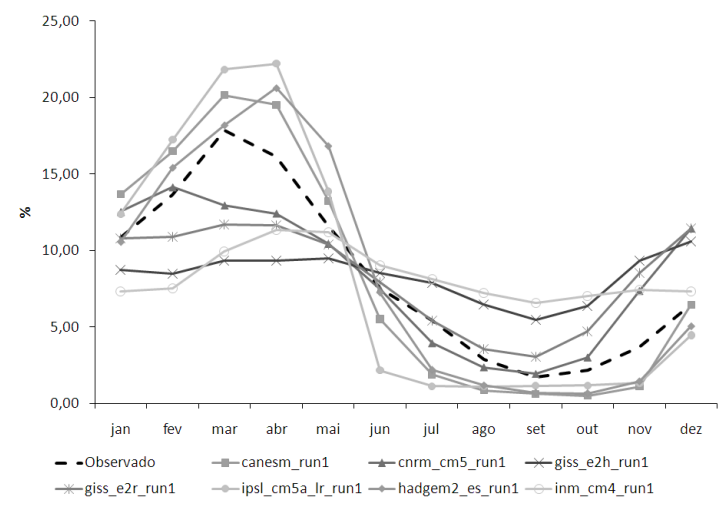

b)

Figura 4 - Climatologia dos Modelos do CMIP5 para século XX (1901-1999) para o Nordeste Setentrional do Brasil: (a) Quantidade de precipitação por mês; (b) Percentual de chuva por mês.

precipitação nos meses da estação chuvosa, enquanto o modelo francês IPSL-CM4 subestima a climatologia da precipitação durante a pré-estação e pós-estação (total anual 21\% abaixo das observações), superestimando a contribuição percentual das chuvas entre janeiro e maio. O modelo CNRM_CM5 superestima a precipitação observada nos meses de préestação, indicando uma contribuição percentual no trimestre novembro, dezembro e janeiro cerca de $10 \%$ maior do que nas observações.

Na Tabela 2, são mostrados os índices estatísticos da avaliação dos modelos do CMIP5 em ordem de classificação para região do Nordeste Setentrional do Brasil, para igual a 1/3. As rodadas r2i1p1, r3i1p1 e r1i1p1 do modelo CanESM, nessa ordem, apresentaram o maior valor de $\mathrm{AVAL}_{\mathrm{t}}$ para região do Nordeste Setentrional do Brasil, sugerindo que este modelo possui a melhor representação dos padrões de variações da região.

Quanto à avaliação sazonal, a maioria dos modelos indicou correlações superiores a 0,85 e erro quadrático inferior a $5 \%$ em relação ao percentual de chuva mensal. O modelo canadense CanESM apresentou correlações superiores a 0,97 e RMSE_PC inferior a 2,4\%, o que o classifica como o melhor modelo para região Nordeste Setentrional do Brasil, segundo a avaliação sazonal proposta neste trabalho (usando igual a 0,5), seguido pelo modelo HADGEM2_ES. Os modelos GISS_E2H e INM_CM4 apresentaram correlações inferiores a 0,75 , bem abaixo dos demais modelos, e os maiores RMSE_PC para todas as rodadas. Isto levou esses modelos a apresentarem AVAL inferior a 0,30 , indicando que os mesmos não representam adequadamente a sazonalidade da precipitação no Nordeste Setentrional Brasileiro.

Quanto à avaliação interanual, a maioria dos modelos apresenta correlações superiores a 0,70, indicando uma boa representação dos padrões de variação nessa escala, sendo o modelo INCM4_r1ilp1 o que obteve os maiores valores de AVALa, seguido pelo modelo GISS_E2R_r5ilp1.

Quanto à avaliação interdecadal, a maioria dos modelos indicou correlações negativas, evidenciando sua dificuldade em representar as frequências mais baixas de variação da série. O modelo CANESM_r2i1p1 apresentou o menor DIST associado a uma correlação de 0,371 , por isso obteve o maior AVAL interanual.

\subsubsection{Região Amazônica}

A Figura 5 mostra as climatologias dos modelos do CMIP5 (apenas a rodada 1) em comparação com o observado sobre a região da Amazônia, conforme indicado na grade 2 da Figura 1. Os modelos divergem quanto à quantidade de chuva nessa região.

Os modelos CANESM, CNRM_CM5 e IPSL_CM5A_ LR subestimam a precipitação climatológica em todos os meses, resultando em totais anuais respectivamente 56\%, 33\% e $60 \%$ abaixo do observado. Além disso, o IPSL_CM5A_LR apresenta erros na representação dos padrões de variação sazonal, superestimando bastante a contribuição percentual das chuvas no primeiro semestre para o total anual e subestimando-a no segundo.

O modelo HADGEM2_ES subestima a precipitação observada no primeiro quadrimestre do ano e superestima nos demais meses, mostrando erro de fase na representação da sazonalidade, enquanto os modelos GISS_E2R e GISS_E2H superestimam a precipitação climatológica no primeiro e último trimestre do ano, porém subestimam nos demais períodos, apresentando também um pequeno erro de fase.

Na Tabela 3 são mostrados os índices estatísticos dos modelos do CMIP5 em ordem de classificação para região Amazônica, para igual a 1/3. 
Tabela 2 - Modelos do CMIP5, CORREL, RMSE_PC, RMSE, AVAL e classificação para $\alpha \mathrm{c}=\alpha \mathrm{r}=0,5$ para o Nordeste Setentrional do Brasil.

\begin{tabular}{|c|c|c|c|c|c|c|c|c|c|c|c|}
\hline \multirow{2}{*}{ Models } & \multicolumn{3}{|c|}{ Sazonal } & \multicolumn{3}{|c|}{ Interanual } & \multicolumn{3}{|c|}{ Interdecadal } & \multirow{2}{*}{$\begin{array}{l}\text { Total } \\
\text { Avalt }\end{array}$} & \multirow{2}{*}{ Clas } \\
\hline & RMSE_PC & Correl & Avals & Correl & DIST & Avala & Correl & DIST & Avald & & \\
\hline canesm_r2i1p1 & 2,290 & 0,987 & 0,992 & 0,903 & 1,240 & 0,588 & 0,371 & 0,838 & 0,851 & 0,810 & 1 \\
\hline canesm_r3i1p1 & 2,386 & 0,986 & 0,972 & 0,950 & 1,154 & 0,731 & 0,231 & 0,978 & 0,681 & 0,795 & 2 \\
\hline canesm_r1i1p1 & 2,352 & 0,986 & 0,979 & 0,933 & 1,099 & 0,771 & 0,682 & 1,446 & 0,500 & 0,750 & 3 \\
\hline giss_e2r_r5i1p1 & 3,257 & 0,825 & 0,606 & 0,871 & 0,994 & 0,817 & 0,308 & 0,955 & 0,732 & 0,718 & 4 \\
\hline cnrm_cm5_r1i1p1 & 2,642 & 0,871 & 0,786 & 0,737 & 0,904 & 0,769 & $-0,141$ & 1,060 & 0,462 & 0,672 & 5 \\
\hline canesm_r5i1p1 & 2,254 & 0,987 & 1,000 & 0,807 & 0,998 & 0,743 & $-0,387$ & 1,198 & 0,251 & 0,665 & 6 \\
\hline hadgem2_es_r1i1p1 & 2,494 & 0,970 & 0,931 & 0,836 & 1,014 & 0,758 & $-0,521$ & 1,123 & 0,255 & 0,648 & 7 \\
\hline giss_e2r_r2i1p1 & 3,210 & 0,832 & 0,624 & 0,763 & 1,077 & 0,611 & 0,478 & 1,082 & 0,702 & 0,646 & 8 \\
\hline ipsl_cm5a_lr_r4i1p1 & 3,284 & 0,968 & 0,768 & 0,820 & 1,119 & 0,627 & 0,447 & 1,350 & 0,478 & 0,625 & 9 \\
\hline giss_e2r_r1i1p1 & 3,215 & 0,834 & 0,626 & 0,929 & 1,191 & 0,669 & $-0,154$ & 1,005 & 0,500 & 0,599 & 10 \\
\hline ipsl_cm5a_lr_r2i1p1 & 3,387 & 0,966 & 0,745 & 0,856 & 1,008 & 0,786 & $-0,514$ & 1,130 & 0,252 & 0,594 & 11 \\
\hline ipsl_cm5a_lr_r1i1p1 & 3,342 & 0,966 & 0,755 & 0,800 & 1,082 & 0,645 & $-0,303$ & 1,153 & 0,322 & 0,574 & 12 \\
\hline giss_e2r_r3i1p1 & 3,263 & 0,827 & 0,608 & 0,913 & 1,351 & 0,480 & $-0,209$ & 0,812 & 0,630 & 0,573 & 13 \\
\hline canesm_r4i1p1 & 2,311 & 0,987 & 0,988 & 0,489 & 1,052 & 0,341 & 0,012 & 1,258 & 0,370 & 0,566 & 14 \\
\hline Incm4_r1i1p1 & 4,340 & 0,725 & 0,270 & 0,846 & 0,924 & 0,865 & $-0,122$ & 1,154 & 0,396 & 0,510 & 15 \\
\hline giss_e2r_r4i1p1 & 3,207 & 0,835 & 0,629 & 0,673 & 1,168 & 0,416 & $-0,297$ & 0,988 & 0,455 & 0,500 & 16 \\
\hline ipsl_cm5a_lr_r3i1p1 & 3,362 & 0,965 & 0,749 & 0,705 & 1,369 & 0,235 & $-0,482$ & 0,908 & 0,441 & 0,475 & 17 \\
\hline giss_e2h_r4i1p1 & 4,604 & 0,622 & 0,096 & 0,782 & 0,945 & 0,773 & 0,330 & 1,237 & 0,519 & 0,462 & 18 \\
\hline giss_e2h_r1i1p1 & 4,610 & 0,608 & 0,079 & 0,801 & 1,001 & 0,733 & $-0,214$ & 0,905 & 0,554 & 0,455 & 19 \\
\hline giss_e2h_r3i1p1 & 4,613 & 0,619 & 0,090 & 0,692 & 0,904 & 0,719 & $-0,345$ & 0,853 & 0,541 & 0,450 & 20 \\
\hline giss_e2h_r5i1p1 & 4,597 & 0,629 & 0,105 & 0,760 & 0,986 & 0,705 & $-0,178$ & 1,145 & 0,380 & 0,396 & 21 \\
\hline giss_e2h_r2i1p1 & 4,717 & 0,560 & 0,000 & 0,676 & 1,033 & 0,564 & $-0,146$ & 0,873 & 0,608 & 0,391 & 22 \\
\hline
\end{tabular}

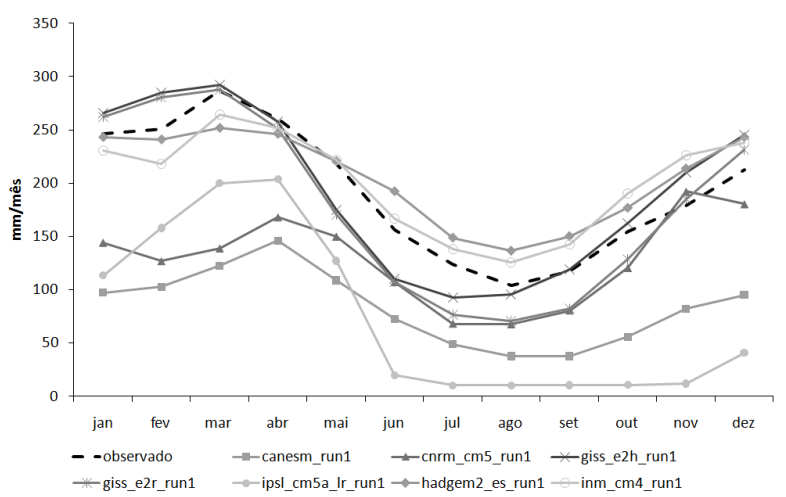

a)

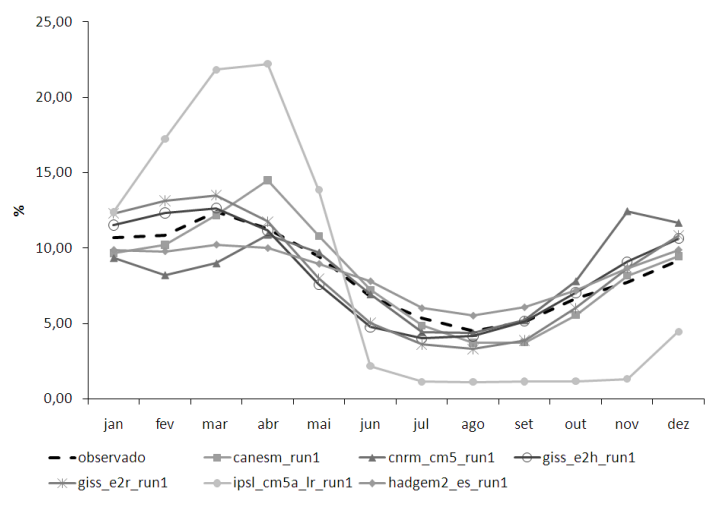

b)

Figura 5 - Climatologia dos Modelos do CMIP5 para século XX (1901-1999) para a Região Amazônica: (a) Quantidade de precipitação por mês; (b) Percentual de chuva por mês.

Quanto à avaliação sazonal, o modelo HADGEM2_ES apresentou o maior AVAL para região amazônica, porém, os modelos CANESM, GISS_E2R, e GISS_E2H também apresentaram AVAL superiores a 0,90. O modelo CNRM_CM5 apresentou correlação de aproximadamente 0,70 , mostrando-se inferior aos demais na representação da sazonalidade nessa região, enquanto o modelo IPSL_CM5A_LR, apesar de apresentar correlação superior a 0,90, indicou RMSE_PC ligeiramente superior aos demais, o que o levou a ser classificado entre os piores modelos na representação da sazonalidade na região Amazônica. A avaliação geral apontou algumas rodadas dos modelos GISS_E2H e GISS_E2R como melhores representações dos padrões de variação na região amazônica.

\subsubsection{Bacia do Prata}

A Figura 6 mostra as climatologias dos modelos do CMIP5 (apenas a rodada 1 de cada modelo) em comparação com o observado sobre a região da bacia do Prata, conforme indicado na grade 3 da Figura 1. Os modelos representam bem a climatologia observada, o que fica evidente na Figura 3a e nas correlações mostradas na Tabela 4.

O modelo HADGEM2_ES superestima a precipitação climatológica em todos os meses, resultando em um total anual 30\% acima do observado. Além disso, apresenta erros na distribuição sazonal da precipitação, subestimando a contribuição percentual das chuvas no primeiro e último 
Tabela 3 - Modelos do CMIP5, CORREL, RMSE_PC, RMSE, AVAL e classificação dos para $\alpha c=\alpha r=0,5$ para a Região Amazônica.

\begin{tabular}{|c|c|c|c|c|c|c|c|c|c|c|c|}
\hline \multirow{2}{*}{ Models } & \multicolumn{3}{|c|}{ Sazonal } & \multicolumn{3}{|c|}{ Interanual } & \multicolumn{3}{|c|}{ Interdecadal } & \multirow{2}{*}{ Avalt } & \multirow{2}{*}{ Clas } \\
\hline & RMSE_PC & Correl & Avals & Correl & DIST & Aval & Correl & DIST & Aval & & \\
\hline giss_e2h_r1i1p1 & 1,172 & 0,938 & 0,927 & 0,892 & 0,012 & 0,921 & 0,579 & 0,620 & 0,530 & 0,792 & 1 \\
\hline giss_e2r_r3i1p1 & 1,408 & 0,966 & 0,950 & 0,948 & 0,060 & 0,920 & $-0,283$ & 0,897 & 0,360 & 0,743 & 2 \\
\hline giss_e2h_r2i1p1 & 1,224 & 0,933 & 0,914 & 0,891 & 0,071 & 0,826 & $-0,356$ & 1,210 & 0,466 & 0,735 & 3 \\
\hline giss_e2r_r2i1p1 & 1,494 & 0,966 & 0,942 & 0,787 & 0,017 & 0,772 & $-0,389$ & 1,027 & 0,378 & 0,697 & 4 \\
\hline ipsl_cm5a_lr_r2i1p1 & 2,396 & 0,972 & 0,860 & 0,899 & 0,039 & 0,888 & $-0,637$ & 1,091 & 0,323 & 0,690 & 5 \\
\hline giss_e2r_r5i1p1 & 1,458 & 0,963 & 0,941 & 0,799 & 0,083 & 0,684 & 0,305 & 0,565 & 0,416 & 0,681 & 6 \\
\hline giss_e2r_r1i1p1 & 1,427 & 0,966 & 0,949 & 0,864 & 0,193 & 0,600 & 0,355 & 0,679 & 0,481 & 0,676 & 7 \\
\hline canesm_r5i1p1 & 1,325 & 0,941 & 0,917 & 0,897 & 0,099 & 0,792 & 0,062 & 0,505 & 0,311 & 0,673 & 8 \\
\hline giss_e2h_r5i1p1 & 1,179 & 0,938 & 0,927 & 0,741 & 0,138 & 0,522 & $-0,037$ & 1,204 & 0,569 & 0,673 & 9 \\
\hline Incm4_r1i1p1 & 1,053 & 0,934 & 0,931 & 0,951 & 0,142 & 0,796 & $-0,729$ & 1,049 & 0,275 & 0,667 & 10 \\
\hline giss_e2h_r3i1p1 & 1,223 & 0,932 & 0,912 & 0,827 & 0,182 & 0,568 & 0,351 & 0,519 & 0,412 & 0,631 & 11 \\
\hline canesm_r4i1p1 & 1,187 & 0,954 & 0,953 & 0,653 & 0,144 & 0,396 & $-0,597$ & 1,590 & 0,544 & 0,631 & 12 \\
\hline canesm_r2i1p1 & 1,298 & 0,946 & 0,929 & 0,783 & 0,292 & 0,337 & 0,777 & 0,550 & 0,567 & 0,611 & 13 \\
\hline hadgem2_es_r1i1p1 & 1,070 & 0,960 & 0,975 & 0,728 & 0,079 & 0,597 & $-0,628$ & 0,922 & 0,255 & 0,609 & 14 \\
\hline canesm_r1i1p1 & 1,228 & 0,941 & 0,926 & 0,651 & 0,139 & 0,400 & 0,702 & 0,390 & 0,475 & 0,600 & 15 \\
\hline$\underset{\text { r4i1p1 }}{\text { ipsl_cm5a_lr }}$ & 2,280 & 0,972 & 0,872 & 0,576 & 0,199 & 0,206 & 0,259 & 1,285 & 0,701 & 0,593 & 16 \\
\hline cnrm_cm5_r1i1p1 & 2,068 & 0,679 & 0,399 & 0,877 & 0,050 & 0,841 & 0,692 & 0,508 & 0,521 & 0,587 & 17 \\
\hline giss_e2r_r4i1p1 & 1,427 & 0,968 & 0,953 & 0,634 & 0,152 & 0,357 & $-0,431$ & 0,947 & 0,331 & 0,547 & 18 \\
\hline canesm_r3i1p1 & 1,443 & 0,939 & 0,902 & 0,885 & 0,331 & 0,411 & $-0,301$ & 0,834 & 0,327 & 0,547 & 19 \\
\hline $\begin{array}{c}\text { ipsl_cm5a_lr_ } \\
\text { r3i1p1 }\end{array}$ & 2,447 & 0,975 & 0,860 & 0,815 & 0,169 & 0,571 & $-0,643$ & 0,795 & 0,197 & 0,543 & 20 \\
\hline giss_e2h_r4i1p1 & 1,200 & 0,936 & 0,921 & 0,601 & 0,183 & 0,266 & $-0,482$ & 1,083 & 0,371 & 0,519 & 21 \\
\hline $\begin{array}{c}\text { ipsl_cm5a_lr_ } \\
\text { r1i1p1 }\end{array}$ & 5,996 & 0,905 & 0,382 & 0,875 & 0,067 & 0,812 & 0,136 & 0,420 & 0,300 & 0,498 & 22 \\
\hline
\end{tabular}

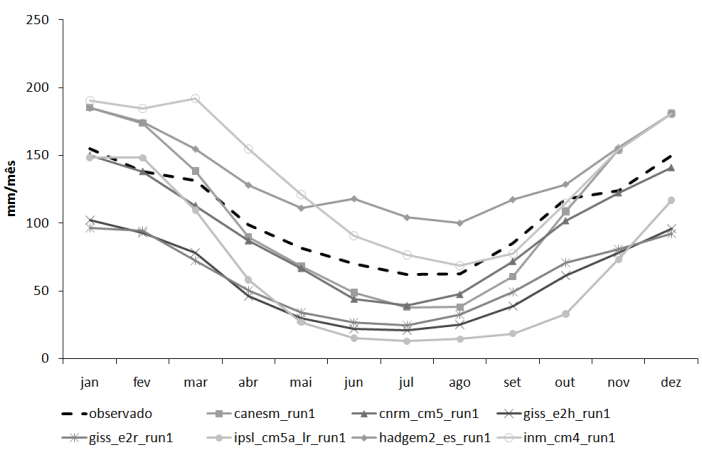

a)

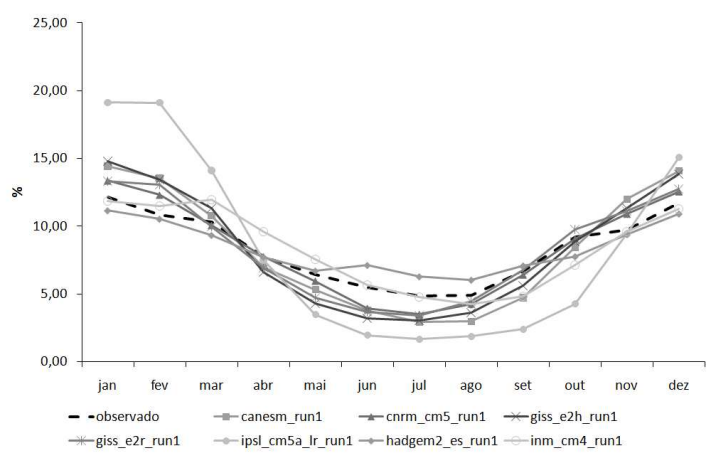

b)

Figura 6 - Climatologia dos Modelos do CMIP5 para século XX (1901-1999) para bacia do Prata: (a) Quantidade de precipitação por mês; (b) Percentual de chuva por mês.

trimestre do ano para o total anual e superestimando-a nos demais períodos do ano. Os modelos GISS_E2R, GISS_E2H e IPSL_CM5_LR subestimam a precipitação climatológica em todos os meses, resultando em totais anuais respectivamente $46 \%, 43 \%$ e $39 \%$ abaixo do observado. Além disso, o modelo IPSL_CM5A_IR apresenta erros de fase na distribuição anual da precipitação, superestimando a contribuição percentual das chuvas nos meses de dezembro, janeiro, fevereiro e março e subestimando-a nos demais meses.

$\mathrm{Na}$ Tabela 4 são mostrados os índices estatísticos RMSE_PC, CORREL e AVAL dos modelos do CMIP5 em ordem de classificação para bacia do Prata, para $\alpha_{c}$ igual a 0,5 .
Todos os modelos indicaram correlações superiores a 0,90 e erro quadrático inferior a $5 \%$ em relação ao percentual de chuva mensal. O modelo CNRM_CM5 apresentou correlação superior a 0,99 e RMSE_PC inferior a 1\%, o que o classifica como o melhor modelo para região da bacia do Prata, segundo a avaliação proposta neste trabalho (usando igual a 0,5), seguido pelos modelos GISS_E2R e GISS_E2H. O modelo IPSL_CM5A apresentou correlação ligeiramente inferior aos demais modelos, associada a um RMSE_PC elevado (superior a 4\%). Isto levou esse modelo a apresentar AVAL aproximadamente igual a 0 (zero), indicando que o mesmo possui maior dificuldade de representar a sazonalidade da precipitação na bacia do 
Tabela 4 - Modelos do CMIP5, CORREL, RMSE_PC, RMSE, AVAL e classificação para $\alpha \mathrm{c}=\alpha \mathrm{r}=0,5$ para a bacia do Prata.

\begin{tabular}{|c|c|c|c|c|c|c|c|c|c|c|c|}
\hline \multirow{2}{*}{ Models } & \multicolumn{3}{|c|}{ Sazonal } & \multicolumn{3}{|c|}{ Interanual } & \multicolumn{3}{|c|}{ Interdecadal } & \multirow{2}{*}{ Avalt } & \multirow{2}{*}{ Clas } \\
\hline & RMSE_PC & Correl & Avals & Correl & DIST & Aval & Correl & DIST & Aval & & \\
\hline cnrm_cm5_r1i1p1 & 0,946 & 0,990 & 0,992 & 0,873 & 0,191 & 0,703 & 0,312 & 0,301 & 0,877 & 0,857 & 1 \\
\hline giss_e2h_r2i1p1 & 1,735 & 0,989 & 0,870 & 0,885 & 0,197 & 0,711 & 0,456 & 0,160 & 0,979 & 0,853 & 2 \\
\hline giss_e2r_r4i1p1 & 1,273 & 0,980 & 0,873 & 0,797 & 0,019 & 0,794 & 0,281 & 0,627 & 0,752 & 0,806 & 3 \\
\hline canesm_r4i1p1 & 1,887 & 0,983 & 0,807 & 0,712 & 0,099 & 0,596 & 0,513 & 0,343 & 0,936 & 0,780 & 4 \\
\hline giss_e2r_r5i1p1 & 1,225 & 0,981 & 0,889 & 0,712 & 0,060 & 0,639 & 0,466 & 0,661 & 0,809 & 0,779 & 5 \\
\hline giss_e2h_r5i1p1 & 1,819 & 0,991 & 0,872 & 0,749 & 0,070 & 0,676 & 0,295 & 0,539 & 0,788 & 0,779 & 6 \\
\hline giss_e2h_r4i1p1 & 1,694 & 0,985 & 0,849 & 0,727 & 0,053 & 0,666 & 0,150 & 0,389 & 0,787 & 0,767 & 7 \\
\hline hadgem2_es_r1i1p1 & 0,965 & 0,964 & 0,814 & 0,664 & 0,006 & 0,635 & 0,334 & 0,729 & 0,736 & 0,729 & 8 \\
\hline canesm_r1i1p1 & 1,817 & 0,985 & 0,827 & 0,721 & 0,059 & 0,651 & $-0,011$ & 0,611 & 0,651 & 0,709 & 9 \\
\hline canesm_r2i1p1 & 1,916 & 0,985 & 0,813 & 0,919 & 0,345 & 0,595 & 0,328 & 0,814 & 0,705 & 0,704 & 10 \\
\hline giss_e2r_r1i1p1 & 1,246 & 0,979 & 0,870 & 0,682 & 0,151 & 0,500 & 0,027 & 0,420 & 0,732 & 0,701 & 11 \\
\hline canesm_r3i1p1 & 1,998 & 0,981 & 0,778 & 0,765 & 0,328 & 0,414 & 0,240 & 0,260 & 0,865 & 0,686 & 12 \\
\hline giss_e2h_r3i1p1 & 1,716 & 0,989 & 0,874 & 0,568 & 0,026 & 0,489 & $-0,129$ & 0,509 & 0,643 & 0,669 & 13 \\
\hline giss_e2r_r2i1p1 & 1,234 & 0,988 & 0,934 & 0,673 & 0,134 & 0,507 & $-0,816$ & 0,454 & 0,410 & 0,617 & 14 \\
\hline giss_e2r_r3i1p1 & 1,227 & 0,976 & 0,857 & 0,683 & 0,114 & 0,541 & $-0,839$ & 0,588 & 0,355 & 0,584 & 15 \\
\hline canesm_r5i1p1 & 1,897 & 0,985 & 0,819 & 0,559 & 0,025 & 0,480 & $-0,850$ & 0,542 & 0,367 & 0,555 & 16 \\
\hline giss_e2h_r1i1p1 & 1,801 & 0,989 & 0,857 & 0,661 & 0,172 & 0,450 & $-0,836$ & 0,852 & 0,265 & 0,524 & 17 \\
\hline ipsl_cm5a_Ir_r4i1p1 & 4,357 & 0,918 & 0,006 & 0,945 & 0,231 & 0,753 & 0,109 & 0,452 & 0,750 & 0,503 & 18 \\
\hline ipsl_cm5a_Ir_r3i1p1 & 4,334 & 0,917 & 0,003 & 0,788 & 0,011 & 0,791 & 0,074 & 0,570 & 0,696 & 0,497 & 19 \\
\hline ipsl_cm5a_Ir_r2i1p1 & 4,338 & 0,920 & 0,025 & 0,859 & 0,285 & 0,582 & $-0,086$ & 0,417 & 0,691 & 0,433 & 20 \\
\hline Incm4_r1i1p1 & 1,175 & 0,912 & 0,432 & 0,734 & 0,462 & 0,226 & 0,020 & 0,798 & 0,597 & 0,419 & 21 \\
\hline ipsl_cm5a_Ir_r1i1p1 & 4,337 & 0,917 & 0,004 & 0,766 & 0,106 & 0,658 & $-0,353$ & 1,598 & 0,182 & 0,281 & 22 \\
\hline
\end{tabular}

Prata. A rodada ipsl_cm5a_lr__r1i1p1 possui dificuldade de representação dos padrões de variação, tanto na bacia do Prata, quanto na região amazônica, sendo o pior classificado, segundo a avaliação proposta nesse trabalho, nas duas regiões. Em contraste, o modelo CNRM_CM5_r1i1p1 apresentou a melhor a avaliação geral para o bacia do Prata.

\subsection{Projeções}

A Figura 7 apresenta as anomalias sazonais da precipitação para o Nordeste Setentrional do Brasil (a), região Amazônica (b) e bacia do Prata (c), respectivamente, para os modelos cujas projeções para o cenário RCP8.5 para o restante do século XXI já se encontravam disponíveis quando da preparação do presente trabalho.

Nas três regiões, os modelos apresentaram divergências nas anomalias da precipitação, durante todo o ano. No Nordeste do Brasil, a maioria dos modelos indica aumento de precipitação no primeiro quadrimestre de até $30 \%$ por mês, exceto o modelo canesm_r1i1p1 (melhor modelos segundo a avaliação geral), que apresentou reduções de aproximadamente $10 \%$ em cada mês desse período. Os modelos mostram grande dispersão nos meses de junho, julho e dezembro, indicando possíveis impactos na pré-estação e pós-estação chuvosa na região.

Já na região amazônica, os modelos convergem a maior parte do ano quanto ao sinal da anomalia de precipitação, indicando possível redução de precipitação ao longo de todo o ano. A exceção é o modelo ipsl-cm5a-lr_rli1p1 (pior dos modelos para essa região), que indica aumento na precipitação climatológica na maior parte do segundo semestre.

$\mathrm{Na}$ bacia do Prata, os modelos indicam anomalias sazonais de precipitação positivas durante todo o ano, com exceção de dois modelos (CanESM2_r1i1p1 e ipsl-cm5a-lr r1i1p1). Todos os modelos, com exceção do modelo CanESM2 r1i1p1, durante os meses de janeiro e fevereiro apresentaram anomalias positivas, indicando aumento de até $20 \%$ por mês no regime pluviométrico anual. No segundo semestre o padrão manteve-se, entretanto, os modelos CanESM2_r1i1p1 e o ipslcm5a-lr_r1i1p1, apontaram anomalias negativas entre os meses de julho a novembro.

Na Tabela 5, são mostradas as anomalias dos modelos do CMIP5 para as três regiões do estudo, para os períodos de 2010 a 2039, 2040 a 2069 e 2070 a 2099.

Na região do Nordeste Setentrional do Brasil os modelos cnrm-cm5_r1i1p1, hadgem2-ES_r1i1p1 e o ipsl-cm5a-lr_r1i1p1 mostram anomalias positivas nos três períodos analisados de até $32,5 \%$ no terceiro período, enquanto os demais apresentam anomalias negativas de até $30,6 \%$ no terceiro período.

Na região Amazônica, todos os modelos apresentaram anomalias de precipitação negativas (exceto o modelo ipsl-cm5a-lr_r1ilp1), com reduções de precipitação de até 20,5\%,33,6 e 39,5\% para os períodos de 2010 a 2039, 2040 a 2069 e 2070 a 2099, respectivamente.

Na bacia do Prata os modelos cnrm-cm5_r1i1p1, giss-e2-r_r1i1p1 e o hadgem2-ES_r1i1p1 indicam anomalias 


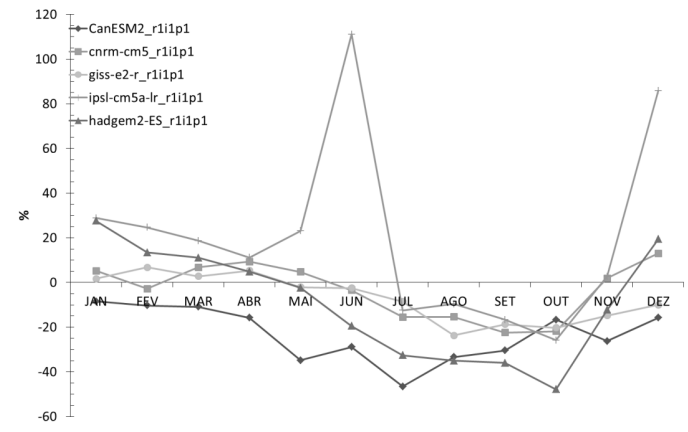

a)

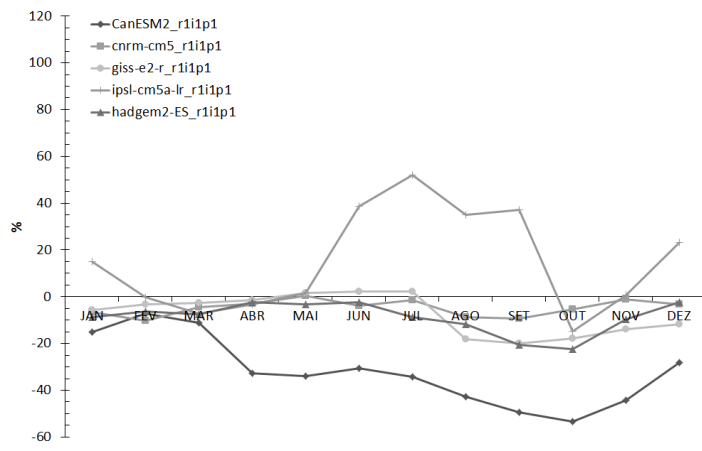

b)

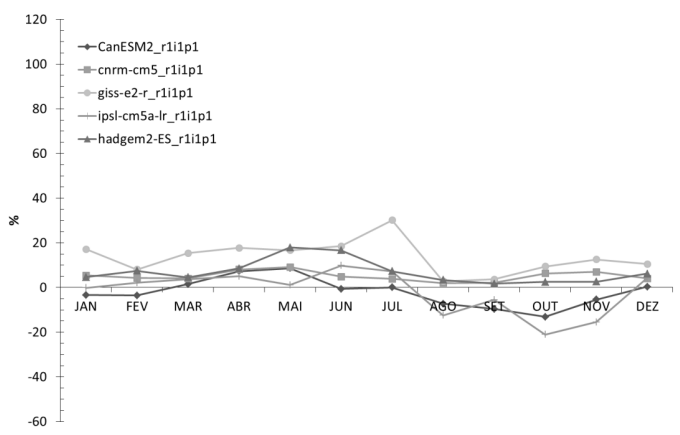

c)

Figura 7 -Anomalia sazonal das precipitações para o século XXI do cenário rcp85: (a)Nordeste do Brasil; (b) região Amazônica e (c) bacia do Prata .

positivas e os demais apresentam anomalias negativas. Nesta região, o impacto na precipitação indicado pelos modelos é inferior ao projetado para as demais.

\section{CONCLUSÕES}

A avaliação proposta neste trabalho identificou os melhores e piores modelos na representação dos padrões de variação da precipitação no século XX para o Nordeste Setentrional do Brasil, Amazônia e bacia do Prata. Essas informações podem ser usadas por cientistas e gestores na análise das projeções de mudanças climáticas sobre essas regiões o século XXI e seus impactos. Em particular, os resultados deste trabalho podem ser utilizados na avaliação do possível impacto das mudanças no clima sobre as vazões e evapotranspiração nessas regiões, utilizando o maior número de modelos possível para tratar e dimensionar a incerteza existente nessas projeções.

Quanto à avaliação sazonal dos modelos do CMIP5 listados, algumas observações são destacadas:

- Os modelos globais do CMIP5 analisados apresentam correlações elevadas em relação à climatologia observada no período de 1901 a 1999 para as regiões do Nordeste Setentrional do Brasil, bacia do Prata e Amazônia, mostrando que os modelos são capazes de capturar os padrões de variações sazonais;
- A avaliação feita com os dados médios apontou o modelo CANESM como aquele com mais altos valores de AVALs para o Nordeste Setentrional Brasileiro, seguido pelo modelo HADGEM2_ES. O modelo CNRM_CM5 apresentou o maior AVAL para região do Prata, seguido pelos modelos GISS_E2R e GISS_E2H. Já para a Amazônia, o modelo HADGEM2_ES é o melhor classificado, segundo o método de avaliação deste trabalho.

- Os modelos apresentaram GISS_E2H e INM_CM4 correlações sazonais ligeiramente inferiores aos demais no Nordeste Setentrional do Brasil. Dessa forma, mostram-se inferiores aos outros modelos do CMIP5 na representação da climatologia média sobre a região.

- O modelo IPSL_CM5A apresenta AVALs aproximadamente igual a 0 (zero) na bacia do Prata, indicando que o mesmo possui dificuldade de representar a sazonalidade nesta região.

- O modelo CNRM_CM5 apresentou correlação sazonal de aproximadamente 0,70 na região amazônica, inferior aos demais na representação da sazonalidade nessa região.

Quanto às avaliações interdecadal e interanual dos modelos IPCC algumas observações são destacadas:

- A maioria dos modelos analisados capta os padrões de variação interanual, com algumas rodadas dos modelos globais 
Tabela 5 - Anomalia das precipitações anuais para as regiões de estudo.

\begin{tabular}{|c|c|c|c|c|}
\hline Região & Modelos do CMIP 5 & 2010-2039 & 2040-2069 & 2070-2099 \\
\hline \multirow{8}{*}{ NEB } & CanESM2_r1i1p1 & $-7,2$ & $-13,4$ & $-30,6$ \\
\hline & CanESM2_r3i1p1 & $-2,3$ & $-10,4$ & $-26,7$ \\
\hline & CanESM2_r4i1p1 & $-8,3$ & $-24,2$ & $-31,0$ \\
\hline & CanESM2_r5i1p1 & $-0,5$ & $-12,8$ & $-22,8$ \\
\hline & cnrm-cm5_r1i1p1 & 2,1 & 2,5 & 2,4 \\
\hline & giss-e2-r_r1i1p1 & $-4,6$ & $-2,9$ & $-3,0$ \\
\hline & hadgem2-ES_r1i1p1 & 7,2 & 3,9 & 6,0 \\
\hline & ipsl-cm5a-Ir_r1i1p1 & 14,2 & 23,3 & 32,5 \\
\hline \multirow{8}{*}{ AMA } & CanESM2_r1i1p1 & $-16,6$ & $-29,9$ & $-38,4$ \\
\hline & CanESM2_r3i1p1 & $-10,6$ & $-27,9$ & $-36,5$ \\
\hline & CanESM2_r4i1p1 & $-20,5$ & $-33,6$ & $-38,5$ \\
\hline & CanESM2_r5i1p1 & $-11,9$ & $-24,9$ & $-39,5$ \\
\hline & cnrm-cm5_r1i1p1 & $-2,6$ & $-4,0$ & $-6,3$ \\
\hline & giss-e2-r_r1i1p1 & $-5,9$ & $-6,2$ & $-6,3$ \\
\hline & hadgem2-ES_r1i1p1 & $-4,3$ & $-5,2$ & $-14,5$ \\
\hline & ipsl-cm5a-Ir_r1i1p1 & 6,4 & 7,0 & 10,1 \\
\hline \multirow{8}{*}{ PRATA } & CanESM2_r1i1p1 & $-3,7$ & $-3,3$ & $-0,1$ \\
\hline & CanESM2_r3i1p1 & $-0,9$ & $-2,7$ & $-3,5$ \\
\hline & CanESM2_r4i1p1 & $-0,8$ & $-3,8$ & 0,1 \\
\hline & CanESM2_r5i1p1 & $-2,0$ & $-2,3$ & $-2,9$ \\
\hline & cnrm-cm5_r1i1p1 & 2,3 & 4,9 & 8,4 \\
\hline & giss-e2-r_r1i1p1 & 5,6 & 12,2 & 19,9 \\
\hline & hadgem2-ES_r1i1p1 & 1,7 & 5,5 & 11,8 \\
\hline & ipsl-cm5a-Ir_r1i1p1 & $-2,7$ & $-0,9$ & 1,9 \\
\hline
\end{tabular}

do IPCC apresentando valores bastante elevados de correlação em relação à precipitação observada durante o século XX. Porém, o mesmo não acontece com a avaliação interdecadal, para a qual a maioria dos modelos mostra muita dificuldade;

- As rodadas do modelo ipsl-cm5a-lr_r1i1p1 não representa apropriadamente a variabilidade plurianual na Amazônia e na bacia do Prata, o mesmo acontecendo com as rodadas do modelo GISS_E2Hem relação ao Nordeste setentrional.

A avaliação geral apontou como melhores simulações do clima presente sobre o Nordeste setentrional as rodadas do modelo CANESM. Para a bacia do Prata as rodadas do modelo francês CNRM_CM5_r1i1p1 apresentaram altos valores de AVALg. Para a Amazônia destacam-se as rodadas do modelo GISS-E2-R_r1ilp1. Estes modelos podem ser considerados como uma boa opção para avançar na investigação dos efeitos das mudanças climáticas sobre os recursos hídricos na América do Sul.

Quanto às projeções dos modelos do CMIP5 analisados para o cenário RCP8.5 do século XXI, há divergências quanto ao futuro da precipitação nas várias regiões da América do Sul. Esse espalhamento pode estar associado à própria incerteza proveniente dos fenômenos meteorológicos que envolvem essa variável (a atmosfera é um sistema caótico, Lorenz, 1965) e/ou má representação dos fenômenos de micro e meso-escala, que precisariam ser resolvidos numa grade de melhor resolução.

- Na região Amazônica, os modelos analisados sugerem maiores possibilidades de redução nas precipitações de até $20,5 \%, 33,6$ e $39,5 \%$ para os períodos de 2010 a 2039, 2040 a 2069 e 2070 a 2099, respectivamente;

- Na região da bacia do Prata, os modelos indicam normalidade no primeiro período e divergem quanto o futuro da precipitação nos períodos seguintes;

- Na região Nordeste do Brasil, a maioria dos modelos analisados mostra impactos maiores nos meses de dezembro, janeiro e fevereiro, porém divergem quanto ao sinal da anomalia;

As divergências dos modelos do CMIP5 analisados quanto às projeções interanuais e sazonais demonstram o alto nível de incerteza existente nessas projeções. Entretanto, estas informações definem uma margem dos possíveis cenários futuros de precipitação do Nordeste Setentrional do Brasil, bacia 
do Prata e região Amazônica, devendo ser usadas para adoção de políticas e gestão em nível de agricultura, recursos hídricos e outras áreas correlacionadas.

Obviamente, projeções com menos incertezas seriam mais interessantes para os tomadores de decisão, no entanto isto não ocorre nas projeções regionais dos modelos do CMIP5, especialmente sobre áreas menores. Artificializar a redução destas incertezas pode induzir estratégias que levem àquilo que, em gerenciamento de risco, se conhece como grandes arrependimentos. Daí, estratégias robustas precisam considerar as incertezas no nível atual de conhecimento.

\section{REFERÊNCIAS}

ALBURQUERQUE,.I.F.; FERREIRA,J.N.; SILVA,M. G. ; DIAS, M. F. Tempo e Clima no Brasil. São Paulo. Oficina de Textos p. 280, 2009.

COMPO,G.P.; J.S. WHITAKER; P.D. SARDESHMUKH: Feasibility of a 100 year reanalysis using only surface pressure data. Bulletin of the American Meteorological Society, v. 87, p. 175-190, 2006

COSTA DOS SANTOS, C. A., BARBOSA DE BRITO, J. I., RAMANA RAO, T. V., ALENCAR MENEZES, H. E., Tendências dos Índices de Precipitação no Estado do Ceará. Revista Brasileira de Meteorologia, v.24, n.1, p. 39-47, 2009.

DUURSMA, E. River flow and temperature profile trends; consequences for water resources. A.de Man, P.E. van Oeveren, S. Montjin, Heineken, N. V. Company. Amsterdam 2002.

GRIMM, A. M.; FERRAZ, S. E.; DOYLE, M. E. Climate variability in southern South America associated with El Nino and La Nina events. Journal of Climate, v. 13, n. 1, p. 35-58, 2000.

HAYLOCK, M., et al. Trends in Total and Extreme South American Rainfall in 1960-2000 and Links with Sea Surface Temperature. Journal of Climate, v.19, n. 8, p. 1490-1512.

IPCC - Intergovernmental Panel on Climate Change: Climate Change 2007: The physical science basis. Cambridge, v. 18 p. 2007.

KANE, R. P. El Niño and La Niña events and rainfall in NE and South Brazil. Revista Brasileira de Geofísica, v.10,p. 49-59, 1992.

LÁZARO,Y. M. C. ;SILVEIRA,C.S. ; SOUZA FILHO, F. A. Avaliação do desempenho dos modelos do IPCC-AR4 no nordeste setentrional do Brasil quanto à variabilidade plurianual da precipitação no século XX. In: IV Simpósio Interncional de Climatologia, 2011, João Pessoa. Mudanças climáticas e seus impactos em áreas urbanas, 2011.
LORENZ, E. N. A study of the predictability of a 28 -Variable Atmospheric Model. Tellus, v.17, n.3, p.321-333, 1965.

MARENGO, J. A.; SOARES, W. R. Impacto das mudanças climáticas no Brasil e Possíveis Cenários Climáticos: Síntese do Terceiro Relatório do IPCC de 2001. CPTECINPE, p. 292005.

MARENGO J.A., VALVERDE, MARIA C. Caracterização do clima no Século XX e Cenário de Mudanças de clima para o Brasil no Século XXI usando os modelos do IPCC-AR4. Revista Multiciência Campinas, v. 8 Maio 2007.

NEW, M., HULME, M., JONES, P.D.,: Representing twentieth century space-time climate variability. Part 1: development of a 1961-90 mean monthly terrestrial climatology. Journal of Climate, v. 12, p. 829-856, 1999.

NEW, M., LISTER, D., HULME, M., MAKIN, I.,: A highresolution data set of surface climate over global land areas. Climate Research , v. 21, p. 1-25, 2001.

NOBRE C. A. Vulnerabilidade, impactos e adaptação à mudança no clima. In: Brasil, Presidência da Republica. Núcleo de Assuntos Estratégicos. Mudança do clima: Negociações Internacionais sobre a Mudança do Clima. Brasília. Núcleo de Assuntos Estratégicos da Presidência da Republica. Secretaria de Comunicação de Governo e Gestão Estratégica. v. 1 parte 2, p. 147-216, 2005.

SANTOS, C. A. C.; BRITO, J. I. B. Análise dos índices de extremos para o semi-árido do Brasil e suas relações com TSM e IVDN. Revista Brasileira de Meteorologia, v. 22, n. 3, p. 303-312, 2007.

SILVEIRA, C.S., SOUZA FILHO, F. A, COSTA, A.A., COUTINHO, M.M., LÁZARO, Y.M.C, SALES, D.C. Avaliação da sazonalidade da precipitação no Nordeste brasileiro simulada pelos modelos do IPCC-AR4. Revista Brasileira de Recursos Hídricos,v, 17 ,n. 3, 2012.

TORRENCE, C., COMPO, G. P., A Practical Guide to Wavelet Analysis. Program in Atmospheric and Oceanic Sciences, University of Colorado, Boulder, Colorado, 1998;

VICTORIA, R.; MARTINELLI, L; MORAES, J.; BALLESTER, M. V.; KRUSHCHE, A. ; PELLEGRINO, G . et al.. Surface air temperature variations in the Amazon region and its border during this century. Journal of Climate, v.11, n.5, p. 1105-1110,1998.

WHITAKER, J.S., G.P.COMPO, X. WEI, AND T.M. HAMILL: Reanalysis without radiosondes using ensemble data assimilation. Montly Weather Review, v, 132, p. 11901200, 2004. 